\title{
Yield Enhancement of Reconfigurable Microfluidics-Based Biochips Using Interstitial Redundancy
}

\author{
FEI SU and KRISHNENDU CHAKRABARTY \\ Duke University
}

\begin{abstract}
Microfluidics-based biochips for biochemical analysis are currently receiving much attention. They automate highly repetitive laboratory procedures by replacing cumbersome equipment with miniaturized and integrated systems. As these microfluidics-based microsystems become more complex, manufacturing yield will have significant influence on production volume and product cost. We propose an interstitial redundancy approach to enhance the yield of biochips that are based on droplet-based digital microfluidics. In this design method, spare cells are placed in the interstitial sites within the microfluidic array, and they replace neighboring faulty cells via local reconfiguration. The proposed design method is evaluated using a set of concurrent real-life bioassays. The defect-tolerant design approach based on space redundancy and local reconfiguration is expected to facilitate yield enhancement of microfluidics-based biochips, especially for the emerging marketplace.
\end{abstract}

Categories and Subject Descriptors: B.7.2 [Integrated Circuits]: Design Aids-Placement and routing; B.8.1 [Performance and Reliability]: Reliability, Testing, and Fault-Tolerance; J.3 [Life and Medical Sciences]—Biology and genetics, health

General Terms: Algorithms, Performance, Design, Reliability

Additional Key Words and Phrases: Microfluidics, yield enhancement, reconfiguration, space redundancy

\section{INTRODUCTION}

Microfluidics-based biochips promise to revolutionize clinical diagnostics, DNA sequencing, and other procedures involving molecular biology [Verpoorte and Rooij 2003]. In contrast to continuous-flow microfluidic biochips consisting of permanently etched micropumps, microvalves, and microchannels [Verpoorte and Rooij 2003], droplet-based microfluidic biochips relying on the manipulation of individual droplets through electrowetting and referred to as digital

The research work was supported by the National Science Foundation under grant number IIS0312352. A preliminary and abridged version of this article was published in Proceedings of Design, Automation and Test in Europe Conference, 2005.

Authors' address: Department of Electrical and Computer Engineering, Duke University, Durham, NC 27708; email: \{fs,krish\}@ee.duke.edu.

Permission to make digital or hard copies of part or all of this work for personal or classroom use is granted without fee provided that copies are not made or distributed for profit or direct commercial advantage and that copies show this notice on the first page or initial screen of a display along with the full citation. Copyrights for components of this work owned by others than ACM must be honored. Abstracting with credit is permitted. To copy otherwise, to republish, to post on servers, to redistribute to lists, or to use any component of this work in other works requires prior specific permission and/or a fee. Permissions may be requested from Publications Dept., ACM, Inc., 1515 Broadway, New York, NY 10036 USA, fax: +1 (212) 869-0481, or permissions@acm.org. (C) 2006 ACM 1550-4832/06/0400-0104 \$5.00

ACM Journal on Emerging Technologies in Computing Systems, Vol. 2, No. 2, April 2006, Pages 104-128. 
microfluidics, have recently been demonstrated [Pollack et al. 2000]. Digital microfluidics offer dynamic reconfigurability, whereby groups of cells in a microfluidic array can be reconfigured to change their functionality during the concurrent execution of a set of bioassays. These features make digital microfluidics a promising platform for massively-parallel DNA analysis, automated drug discovery, and real-time biomolecular recognition.

Future advances in fabrication technology will allow increased integration of microfluidic components in composite microsystems. The 2003 International Technology Roadmap for Semiconductors (ITRS) anticipates that microfluidic biochips will soon be integrated with electronic components in system-on-chip (SOC) design. It is expected that several bioassays will then be concurrently executed in a single microfluidic array [Srinivasan et al. 2003]. However, as in the case of integrated circuits, an increase in density and area of microfluidicsbased biochips will reduce yield, especially for new technology nodes. Low yield is a deterrent to large scale and high-volume production, and it tends to increase production cost. It will take time to ramp up yield-learning based on an understanding of defect types in such mixed-technology SOCs. Therefore, defect-tolerant designs are especially important for the emerging marketplace.

Yield enhancement through space redundancy and reconfiguration has been successfully applied to memories, processor arrays (PAs), and fieldprogrammable gate arrays (FPGAs) [Koren and Koren 1998; Howard et al. 1994]. The success of these techniques can be attributed to the high regularity of memories, PAs, and FPGAs and the ease with which they can be tested and reconfigured to avoid faulty elements. Digital microfluidics-based biochips are also amenable to redundancy-based yield enhancement. As in the case of memories, they contain regular arrays of small elements, and these elements are simple and identical. Similar to FPGAs, reconfigurability is an inherent property of these devices.

In this article, we propose a scheme for incorporating defect tolerance in the design of digital microfluidics-based biochips. While spare rows/columns around a mesh-connected array are often used in fault-tolerant processor arrays and FPGAs [Howard et al. 1994], the property of fluidic locality prevents the application of this simple redundancy technique to microfluidic biochips. Due to the absence of programmable interconnects such as switches between microfluidic cells, a droplet is only able to move directly to the adjacent cells. Thus, a faulty cell can only be replaced by its physically adjacent cells. Consequently, a complicated shifted replacement process is required to utilize the spare cells located in the boundary row/column; this results in an unacceptable increase in the reconfiguration cost.

We propose an interstitial redundancy approach to address this problem. In this approach, spare cells are placed in the interstitial sites within the microfluidic array such that a spare cell can functionally replace any faulty cells that are physically adjacent to it. This defect tolerance method owes its effectiveness to the high utilization of local reconfiguration. We apply this space redundancy technique to a new biochip design with hexagonal electrodes. Microfluidic biochips with different levels of redundancy can be designed to target given yield levels and manufacturing processes. We 
introduce a metric called effective yield to evaluate the yield enhancement provided by these defect-tolerant designs. A set of real-life bioassays, that is, multiplexed in-vitro diagnostics on human physiological fluids, is used to evaluate the proposed method. Simulation results show that the yield of a digital microfluidics-based biochip can be significantly increased with the addition of interstitial redundancy and the use of local reconfiguration.

The organization of the remainder of the article is as follows. In Section 2, we discuss related prior work. Section 3 presents an overview of digital microfluidics-based biochips. It also introduces a new design based on hexagonal electrodes. Section 4 discusses manufacturing defects in digital microfluidic biochips. Section 5 presents a unified test methodology. Reconfiguration techniques for microfluidic biochips are also presented. In Section 6, we introduce various defect-tolerant designs with different levels of redundancy. The defect tolerance of these designs is evaluated in Section 7. In Section 8, multiplexed in-vitro diagnostics on human physiological fluids is used to evaluate the proposed yield improvement methodology. Finally, conclusions are drawn in Section 9.

\section{RELATED PRIOR WORK}

Defect-tolerance techniques have been successfully used for memory chips since the late 1970's [Koren and Koren 1998]. In contrast to memory arrays, few logic circuits have been designed with built-in redundancy. The absence of regularity in these circuits usually leads to high overhead. Regular circuits, such as processor arrays and FPGAs, require less redundancy; a number of defect-tolerance techniques have been proposed to enhance their yield [Howard et al. 1994; Singh 1988].

Microelectromechanical systems (MEMS) is a relatively young field compared to integrated circuits. It employs micromachining techniques, such as surface micromachining and bulk micromachining, in the fabrication process [Madou 1997]. These processes are less mature than standard CMOS manufacturing processes. As a result, the yield for MEMS devices is often less than that for integrated circuits. Attempts have been made in recent years to make MEMS defect tolerant. For example, design-for-manufacturing has been incorporated into the design process for MEMS [Dewey et al. 2000].

Microfluidics differs from MEMS in the underlying energy domains and in the working principles. Hence, defect, tolerance techniques for MEMS cannot be directly applied to microfluidic biochips. Recently a comprehensive costeffective test methodology for digital microfluidics-based biochips was proposed in Su et al. [2003, 2005]. Faults are classified as either manufacturing or operational, and techniques have been developed to detect these faults by electrically controlling and tracking the droplet motion. Based on the detection mechanism, an efficient concurrent testing scheme that interleaves test application with a set of bioassays was also proposed in Su et al. [2004]. These testing techniques can be further integrated with system-level CAD tools to facilitate design-fortest (DFT) and defect tolerance for digital microfluidics-based biochips [Su and Chakrabarty 2005]. 


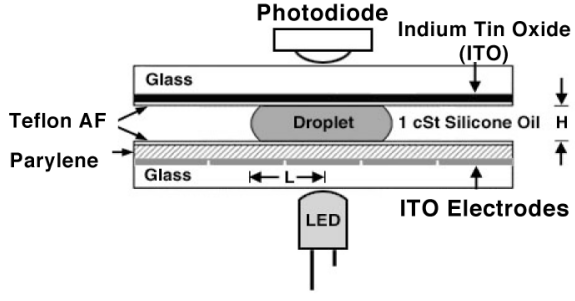

(a)

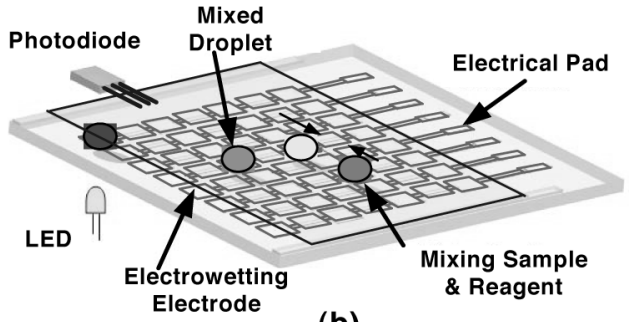

(b)

Fig. 1. (a) Basic cell used in a digital microfluidics-based biochip; (b) top-view of microfluidic array.

\section{DIGITAL MICROFLUIDICS-BASED BIOCHIPS}

Digital microfluidics-based biochips manipulate nanoliter-volume droplets using electrowetting. The basic cell in a digital microfluidics-based biochip consists of two parallel glass plates, shown in Figure 1(a). The bottom plate contains a patterned array of individually controllable electrodes, and the top plate contains a ground electrode. The droplets containing biomedical samples are sandwiched between these two plates and surrounded by a filler medium such as silicone oil. In addition, the droplets are insulated from the electrode array by Parylene C ( $\sim 800 \mathrm{~nm})$, and a thin layer of hydrophobic Teflon AF 1600 $(\sim 50 \mathrm{~nm})$ is coated onto the top and bottom plates to decrease the wettability of the surface.

Electrowetting is the basic principle of microdroplet transportation wherein the interfacial tension of a droplet is modulated with an electric field. A control voltage is applied to an electrode adjacent to the droplet and, at the same time, the electrode just under the droplet is deactivated. Thus, an accumulation of charge in the droplet/insulator interface over the activated electrode results in a surface tension gradient, which consequently causes the transportation of the droplet. By varying the electrical potential along a linear array of electrodes, nanoliter-volume droplets can be transported along this line of electrodes. The velocity of the droplet can be controlled by adjusting the control voltage $(0 \sim 90 \mathrm{~V})$, and droplets have been observed with velocities up to $20 \mathrm{~cm} / \mathrm{s}$ [Pollack et al. 2002]. Furthermore, based on this principle, microdroplets can be transported freely to any location on a two-dimensional array without the need for pumps and valves. The configurations of the microfluidic array are programmed into a microcontroller that controls the voltages of electrodes in the array. A generic digital microfluidics-based biochip consists of a basic microfluidic platform, which moves and mixes droplets containing biochemical samples and reagents, several reservoirs that store and generate the droplets of samples and reagents, and an integrated optical detection system consisting of LEDs and photodiodes (see Figure 1(b)).

In the latest generation of microfluidic biochips, hexagonal electrodes are being used to replace the conventional square electrodes design; this close-packed design is expected to increase the effectiveness of droplet transportation in a $2 \mathrm{D}$ array. The top view of a microfluidic array with hexagonal electrodes is shown in Figure 2(a). A droplet can be moved to an adjacent cell in six possible directions. Recently, printed circuit board (PCB) technology has been used to 


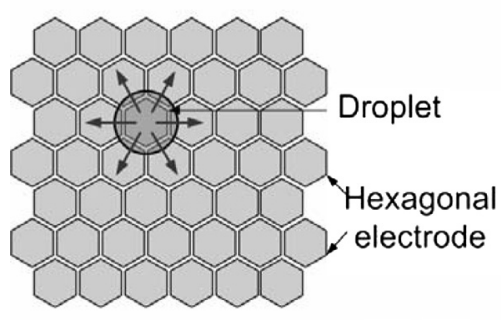

(a)

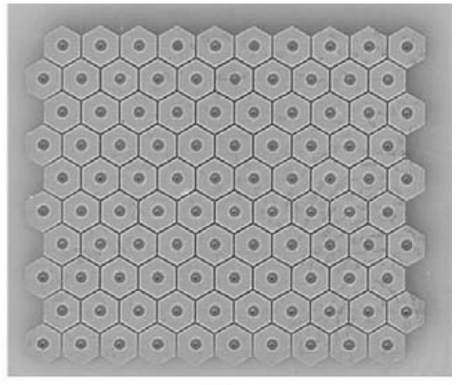

(b)

Fig. 2. (a) Digital microfluidics-based biochip with hexagonal electrodes; (b) fabricated PCB-based microfluidic array.

replace the conventional glass plate as the substrate with the aim of reducing manufacturing cost [Dewey et al. 2000]. Figure 2(b) shows an image of such a fabricated microfluidic array with $10 \times 10$ hexagonal electrodes. In this article, we attempt to make this hexagonal array defect tolerant through space redundancy and local reconfiguration.

\section{MANUFACTURING DEFECTS}

Digital microfluidics-based biochips are fabricated using standard microfabrication techniques, the details of which are described in Pollack [2001]. Microfluidic biochips exhibit behavior resembling that of analog and mixed-signal devices. Therefore, we can classify faults caused by the manufacturing defects as being either catastrophic or parametric, along the lines of fault classification for analog circuits [Jee and Ferguson 1993]. Catastrophic (hard) faults lead to a complete malfunction of the system, while parametric (soft) faults cause a deviation in system performance. A parametric fault is detectable only if this deviation exceeds the tolerance in system performance. However, due to the underlying mixed technology and multiple energy domains, microfluidic biochips exhibit failure mechanisms and defects that are significantly different from failure modes in integrated circuits.

Catastrophic faults may be caused by the following manufacturing defects.

-Dielectric breakdown. The breakdown of the dielectric at high voltage levels creates a short between the droplet and the electrode. When this happens, the droplet undergoes electrolysis preventing further transportation.

- Short between adjacent electrodes. If a short occurs between two adjacent electrodes, the two electrodes shorted effectively form one longer electrode. When a droplet resides on this electrode, it cannot overlap its adjacent electrodes. As a result, the actuation of the droplet can no longer be achieved.

-Degradation of the insulator. This degradation effect is unpredictable and may become apparent gradually during the operation of the microfluidic system. Figure 3 illustrates the electrode degradation due to an insulator degradation defect [Pollack 2001]. A consequence of insulator degradation is that droplets often fragment and their motion is prevented because of the unwanted variation of surface tension forces along their flow path. 


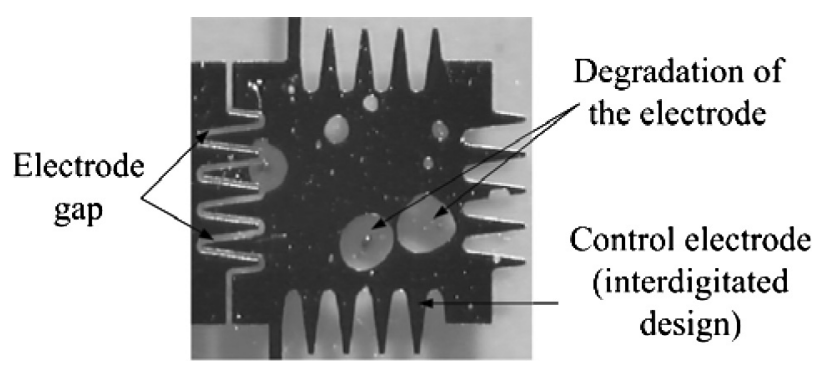

Fig. 3. Top view of a faulty unit cell: electrode degradation.

-Open in the metal connection between the electrode and the control source. This defect results in a failure in activating the electrode for transport.

Manufacturing defects that cause parametric faults include geometrical parameter deviations. The deviation in insulator thickness, electrode length and height between parallel plates may exceed their tolerance value during fabrication.

The level of system integration and the design complexity of digital microfluidics-based biochips are expected to increase in the near future due to the growing need for high-throughput biochemical analysis on a chip. However, shrinking processes, new materials, and the underlying multiple energy domains will make these biochips more susceptible to manufacturing defects. Moreover, some manufacturing defects are expected to be latent, and they may manifest themselves during field operation of the biochips. In addition, harsh operational environments may introduce physical defects such as particle contamination during field operation. Consequently, robust testing techniques are required to ensure system dependability as biochips are deployed for safetycritical applications. Examples of such applications include field diagnostic instruments to monitor infectious diseases and biosensors to detect biochemical toxins and other pathogens.

\section{TESTING AND RECONFIGURATION TECHNIQUES}

In the proposed testing methodology, test stimuli droplets containing the normal conductive fluid (e.g., 0.1M KCL) are released into a two-dimensional microfluidic array from on-chip reservoirs and are guided through the system following the designed testing scheme. Both catastrophic and parametric faults are detected by electrically controlling and tracking the motion of these test stimuli droplets. This testing method is minimally invasive and easy to implement, thus it alleviates the need for expensive and bulky external testing devices.

To facilitate an efficient decision-making process, a unified detection mechanism is needed for both catastrophic and parametric faults [Su et al. 2003]. The proposed unified detection mechanism consists of a simple RC oscillator circuit formed by the sink electrodes and the fluid between them as an insulator; see Figure 4. The capacitance of this structure depends on the presence of the 


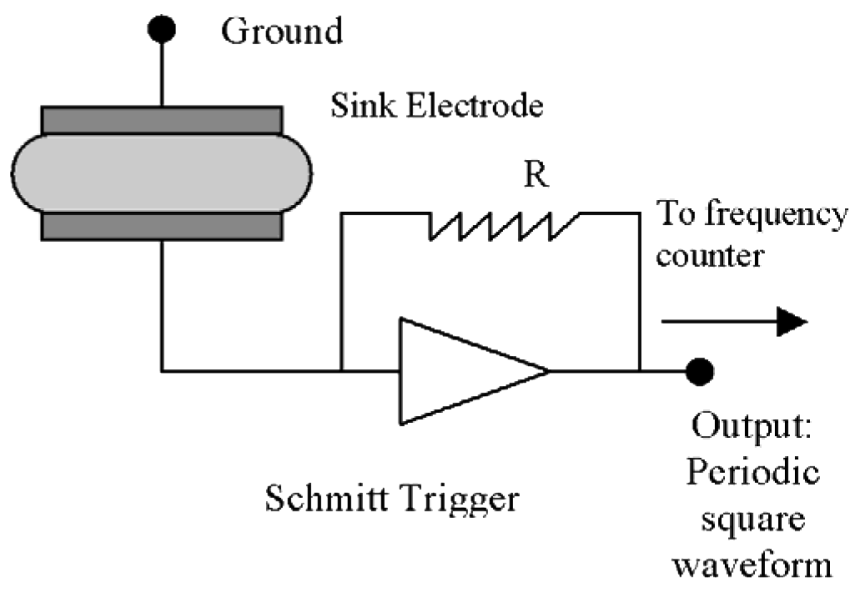

Fig. 4. Simple RC oscillator circuit.

droplet since the filler medium and the droplet have distinct permittivities. By sensing the capacitance of this structure using a simple frequency counter, one can determine whether a droplet has reached the sink. This mechanism can be electronically implemented and easily integrated on-chip. In order to provide a unidirectional and unambiguous detection mechanism, the pass/fail criterion has to be determined based on the presence of the droplet at the sink electrode, and this criterion should be applied for all test cases. In the proposed testing scheme, we associate the fault-free operation with the presence of the droplet at the sink electrode and faulty operation with its absence.

Most catastrophic faults cause a complete cessation of droplet transportation at the system level. Therefore, we can easily detect these faults by using the testing scheme outlined in Figure 5. The fault site in this microfluidic array is highlighted. Droplets are first driven along one direction, for example, along the x-axis, and they are observed at the other end of the array. Each row of the array transports a single droplet of fluid. Due to the catastrophic fault in Row 3, no droplet is observed for this row. As a result, the cells in this row are deemed as candidate faulty cells. Next, droplets are driven following the path along the y-axis, and due to the fault in the array, no droplet is observed at the other end of Column 3. Thus we conclude that the path of Column 3 contains a faulty cell. From the information about the faulty row and column, we can uniquely identify the faulty cell in the array.

This illustration assumes that a catastrophic fault affects only one cell of the array. The testing technique described here can, however, be extended for locating multiple faulty cells, for example, through the use of multistep adaptive fault location methods, and it can also be extended for the detection of parametric faults [Su et al. 2003]. An important advantage of this approach is that it can be integrated into the droplet-manipulation-based microfluidic steps underlying a biochemical reaction, for example, polymerase chain reaction. Concurrent testing can be carried out simultaneously with a bioassay by utilizing unused cells in the array, and a degree of fault tolerance can be 


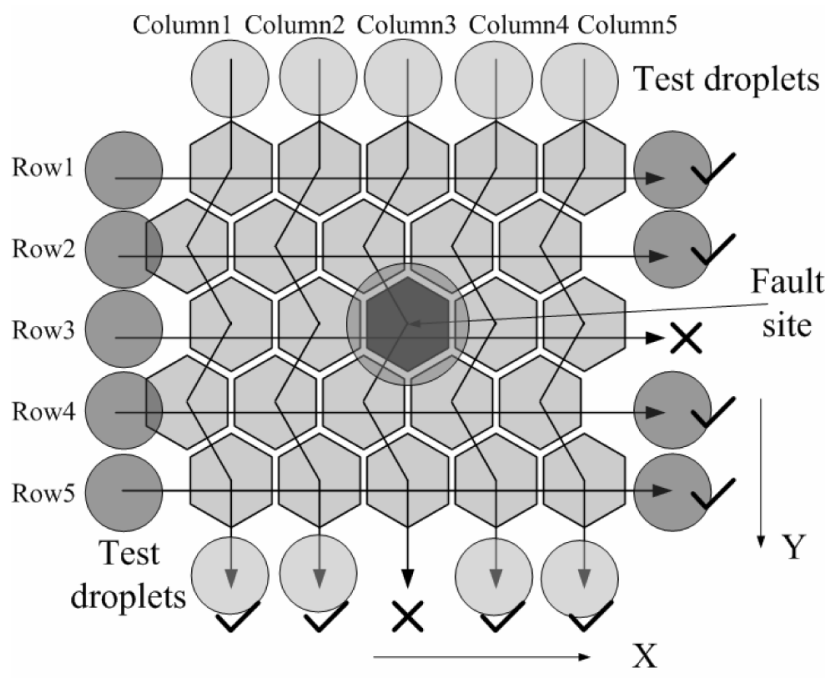

Fig. 5. Illustration of the fault testing scheme.

achieved by reconfiguring the array such that the droplets avoid faulty cells in their flow paths [Su et al. 2004].

The reconfigurability inherent in digital microfluidics-based biochips can be used to increase production yield through production time reconfiguration to bypass manufacturing faults. In this scenario, we assume that a microfluidic biochip has been fabricated along the lines of a reconfigurable array-based design. Defective cells are identified using testing techniques prior to field deployment [Su et al. 2005a, 2005b]. Based on the defect map thus obtained, the configuration of the microfluidic array is changed in such a way that the functionality of the bioassays is not compromised. A similar scheme can be utilized to achieved, longer system lifetime through online reconfiguration to avoid operational faults. Once the concurrent testing procedure determines the faulty status of biochips [Su et al. 2004], the operation of the normal bioassay is stopped. Then reconfiguration techniques are applied to tolerate faults and increase system reliability.

The reconfiguration approaches can be divided into two categories. The first category consists of techniques that do not add space redundancy, that is, spare cells, to the microfluidic array. Instead, they attempt to tolerate the defect by using fault-free unused cells. In order to achieve satisfactory yield using this method, fault tolerance must be considered in the design procedure, for example, in the placement of microfluidic modules in the array [Su and Chakrabarty 2005]. Consequently, it leads to an increase in design complexity. The second category of reconfiguration techniques is application-independent because it incorporates physical redundancy in the microfluidic array. Built-in spare cells can be utilized to replace a defective cell. Since a faulty cell is replaced by a neighboring spare cell, these techniques are also referred to as local reconfiguration. 


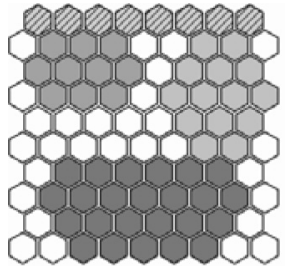

(a)

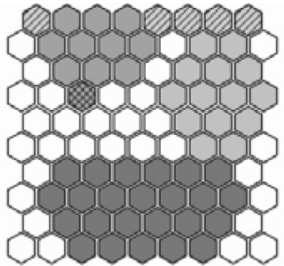

(b)

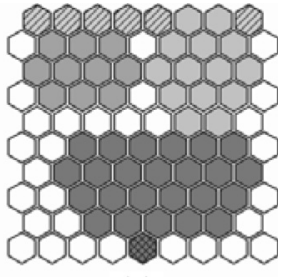

(c)

Spare cells in the boundary row

Faulty cell

Unused cell
Cell in Module 1

Cell in Module 2

Cell in Module 3

Fig. 6. Example of a microfluidic array with one single spare row.

\section{DEFECT-TOLERANT DESIGNS WITH DIFFERENT REDUNDANCY LEVELS}

There are several ways to include spare cells in a defect-tolerant microfluidic array. The first approach is to include spare rows/columns around the microfluidic array. This is a common redundancy technique for PAs and FPGAs. However, in contrast to these electronic arrays with well-defined roles of logic blocks and interconnect, cells in a microfluidic array can be used for storage, transport, or other functional operations on droplets. Due to the absence of separate interconnect entities, droplets can only move to physically adjacent cells. This property is referred to as microfluidic locality. Consequently, the functionality of a faulty cell can only be assumed by its physically-neighboring cells in the array. Microfluidic locality limits the reconfiguration capabilities of the spare rows/columns if they are not adjacent to the faulty cell. In order to utilize the spare cell in the boundary rows/columns, a series of replacement, referred to as shifted replacement is required. In shifted replacement, each faulty cell is replaced by one of its fault-free adjacent cells which is, in turn, replaced by one of its adjacent cells, and so on, until a spare cell from the boundary is incorporated in the reconfigured structure. In many cases, this shifted replacement procedure will not only involve the faulty module, but it will also require the reconfiguration of fault-free modules. Therefore, it significantly increases the complexity of the reconfiguration. Figure 6 shows an example of a microfluidic array with a single spare row. If one cell in Module 1 is faulty, Module 1 can only be relocated to bypass the faulty cell, while other modules remain unchanged; see Figure 6(b). However, if there is one faulty cell in Module 3, the shifted replacement of Module 3 causes the reconfiguration of Module 2 even though it is fault-free; see Figure 6(c).

In order to address the problems resulting from microfluidic locality, a new space redundancy approach termed interstitial redundancy [Singh 1988], is proposed in this article. In this approach, spare cells are located in the interstitial sites within the microfluidic array such that each spare cell is able to functionally replace any one of the primary cells adjacent to it. In contrast to redundancy based on boundary spare rows/columns, interstitial redundancy offers a simple reconfiguration scheme that effectively utilizes local reconfiguration. We apply interstitial redundancy to a digital microfluidics-based biochip with hexagonal 


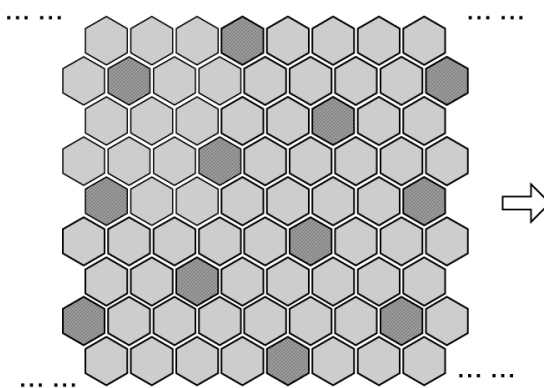

(a)

Spare cell

Primary cell

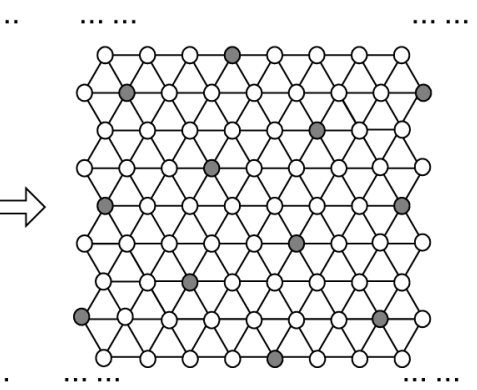

(b)

Node representing spare cell

Node representing primary cell

Fig. 7. Top view and graph model of $\operatorname{DTMB}(1,6)$.

electrodes. Such defect-tolerant microfluidic arrays can incorporate different levels of redundancy depending on the number and location of spare cells. We next introduce some key definitions.

Definition 1. A defect-tolerant design for a digital microfluidics-based biochip, denoted $\operatorname{DTMB}(s, k)$, has interstitial spare cells such that each nonboundary primary cell can be replaced by any one of $s$ spare cells, and each nonboundary spare cell can be used to replace any one of $k$ primary cells.

Definition 2. The redundancy ratio $(R R)$ for a defect-tolerant microfluidic array with interstitial redundancy is the ratio of the number of spare cells in the array to the number of primary cells. Clearly, for a $\operatorname{DTMB}(s, k)$ array of large size, $R R \approx s / k$.

A $\operatorname{DTMB}(1,6)$ design is shown in Figure 7(a). A corresponding graph model, derived from the array, is shown in Figure 7(b). White nodes in the graph represent the primary cells in the microfluidic biochip, while black nodes denote spare cells. An edge between two nodes indicates that the two cells represented by these nodes are physically adjacent in the array. Each primary cell is adjacent to only one spare cell, and every spare cell is adjacent to six primary cells. Therefore, the redundancy ratio for this array approaches 0.1667 as the array size increases.

Other defect-tolerant array designs, for example, $\operatorname{DTMB}(2,6), \operatorname{DTMB}(3,6)$ and $\operatorname{DTMB}(4,4)$, are shown in Figures 8-10. The redundancy ratios of the different designs are listed in Table I.

\section{ESTIMATION OF YIELD ENHANCEMENT}

The effectiveness of various defect-tolerant designs can be determined by estimating their enhanced yields. Here the yield is defined as the percentages of defect-tolerant biochips after manufacturing (including fault-free chips as well as the defective chips that can be successfully reconfigured to avoid defects). The yield analysis in this article is based on the following assumption. 


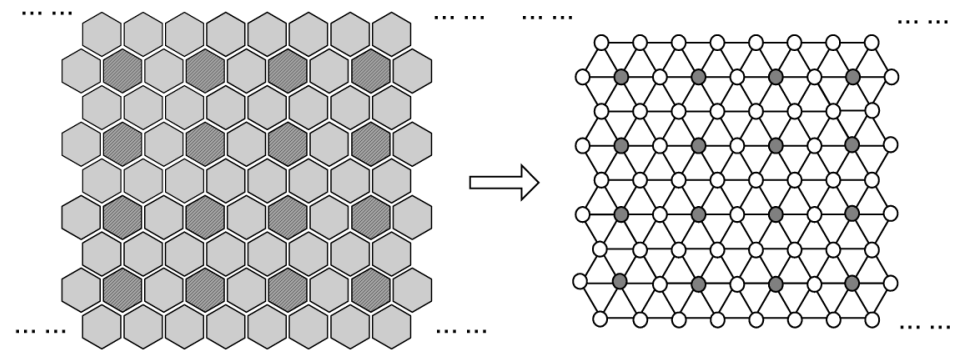

(a) $\operatorname{ADTMB}(2,6)$ design.

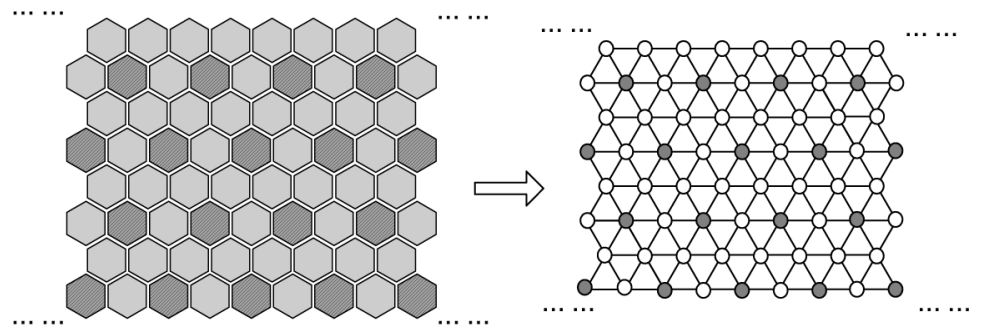

(b) An alternative $D T M B(2,6)$ design.

Fig. 8. $D T M B(2,6)$ designs.

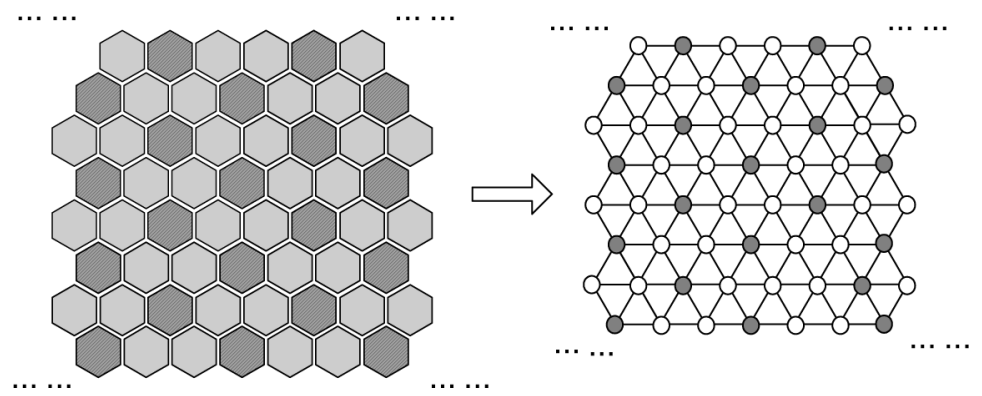

Fig. 9. An $\operatorname{DTMB}(3,6)$ design.

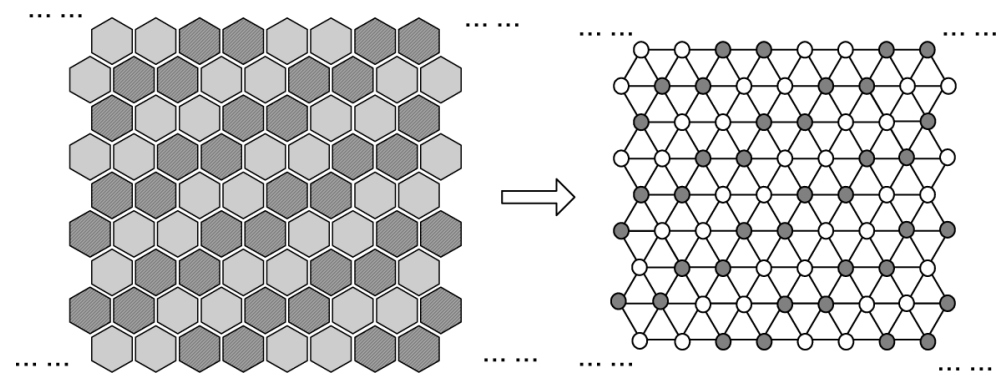

Fig. 10. An FTMB $(4,4)$ design. 
Table I. Redundancy Ratios for Different Defect-Tolerant Architectures

\begin{tabular}{|l|l|l|l|l|}
\hline Design & $D T M B(1,6)$ & $D T M B(2,6)$ & $D T M B(3,6)$ & $D T M B(4,4)$ \\
\hline$R R$ & 0.1667 & 0.3333 & 0.5000 & 1.0000 \\
\hline
\end{tabular}

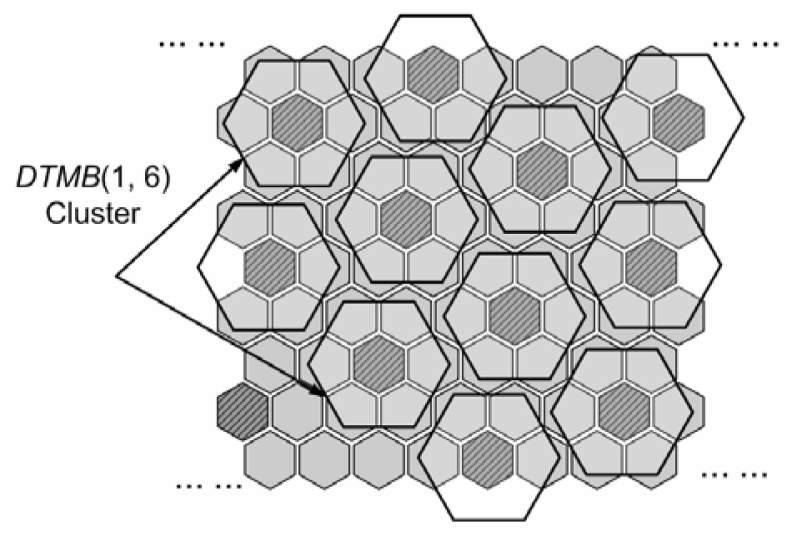

Fig. 11. Illustration of clusters for $\operatorname{DTMB}(1,6)$ array.

Assumption. Each single cell in the microfluidic array, including each primary and spare cell, has the same defect probability $q$. Moreover, the failures of the cells are independent. Let $p=1-q$ denote the survival probability.

Note that the assumption of equal survival probabilities is reasonable since each cell in the microfluidic array has the same structure. In addition, the assumption of independent failures is valid for random and small spot defects which result from imperfect materials and from undesirable chemical and airborne particles.

Based on these assumptions, the yield for a defect-tolerant design can be obtained in terms of $p$. We use both analytical modeling and Monte-Carlo simulation.

Since each primary cell is physically adjacent to only one spare cell in $\operatorname{DTMB}(1,6)$, the spare assignment to a faulty cell is straightforward. Thus, its yield can be easily obtained analytically. We can view $\operatorname{DTMB}(1,6)$ as a composition of identical clusters that consist of one spare cell and six primary cells surrounding the spare cell as shown in Figure 11. The yield $Y_{c}$ of any cluster in $\operatorname{DTMB}(1,6)$ is determined by the likelihood of having at most one failed cell among these seven cells, that is,

$$
Y_{C}=p^{7}+7 p^{6}(1-p)
$$

A biochip with $n$ primary cells can be approximately divided into $n / 6$ clusters. Since the cluster failures are independent, the yield $Y$ for this design is given by

$$
Y_{D T M B(1,6)}=Y_{C}^{n / 6}=\left(p^{7}+7 p^{6}(1-p)\right)^{n / 6} .
$$




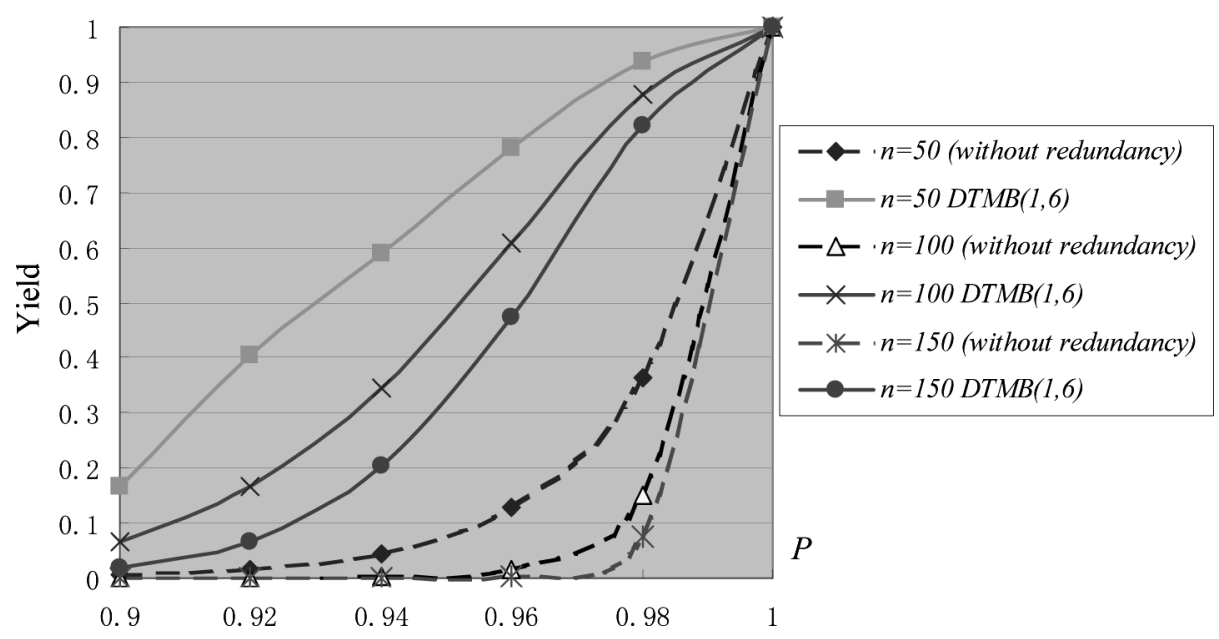

Fig. 12. Estimated yield for $D T M B(1,6)$.

Figure 12 shows the yield for $\operatorname{DTMB}(1,6)$ for different values of $p$ and $n$, and compares it to the yield for a biochip without redundancy. Clearly, interstitial redundancy improves the yield of the microfluidic biochip.

For the defect-tolerant design with a higher level of redundancy such as $\operatorname{DTMB}(2,6)$ and $\operatorname{DTMB}(3,6)$, analytical modeling to determine the yield is not as straightforward as in $\operatorname{DTMB}(1,6)$ due to the more complicated spare assignments. However, we can still apply the cluster-based method in an attempt to estimate the yield of these defect-tolerant array designs. For example, for a $\operatorname{DTMB}(2,6)$ array, we can envision it to consist of identical clusters; each cluster comprises two spare cells and six primary cells following the definition of $\operatorname{DTMB}(2,6)$, that is, each primary cell can be replaced by any one of two spare cells, and each spare cell can be used to replace any one of six primary cells. The graph model of such a cluster in $\operatorname{DTMB}(2,6)$ is shown in Figure 13(a) where an edge between white nodes (representing the primary cells) and black nodes (denoting spare cells) indicates that the primary cell can be replaced by the spare cell. Actually we can view a partition consisting of two neighboring spare cells and their surrounding primary cells in the microfluidic array as a physical representation of the proposed cluster as shown in Figure 13(b). Note that, since the boundary primary cells are shared by two adjacent partitions, we consider only six (i.e., 10/2 + 1) primary cells in a cluster model.

The survival probability of a $\operatorname{DTMB}(2,6)$ cluster is the likelihood of having at most two defective cells among these eight cells, that is,

$$
Y_{C}=p^{8}+8 p^{7}(1-p)+28 p^{6}(1-p)^{2} .
$$

We also assume that a $\operatorname{DTMB}(2,6)$ array with $n$ primary cells can be approximately divided into $n / 6$ clusters, and the failures of the different clusters are independent. The yield $Y$ for this design is then estimated by

$$
Y_{D T M B(2,6)}=Y_{C}^{n / 6}=\left(p^{8}+8 p^{7}(1-p)+28 p^{6}(1-p)^{2}\right)^{n / 6} .
$$




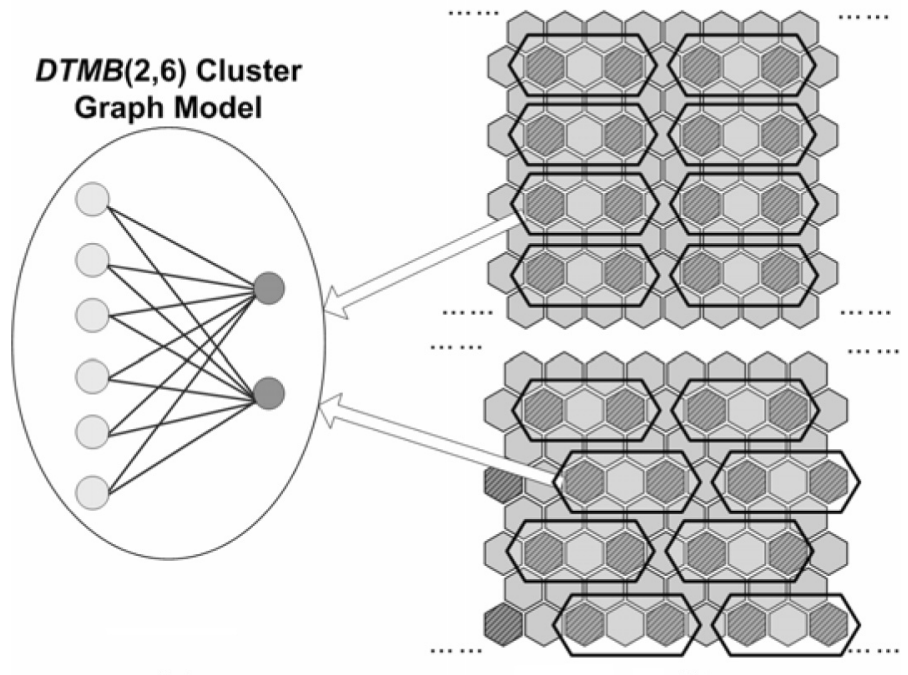

(a)

(b)

Fig. 13. (a) Graph model for a $\operatorname{DTMB}(2,6)$ cluster; (b) illustration of clusters for the $D T M B(2,6)$ array.

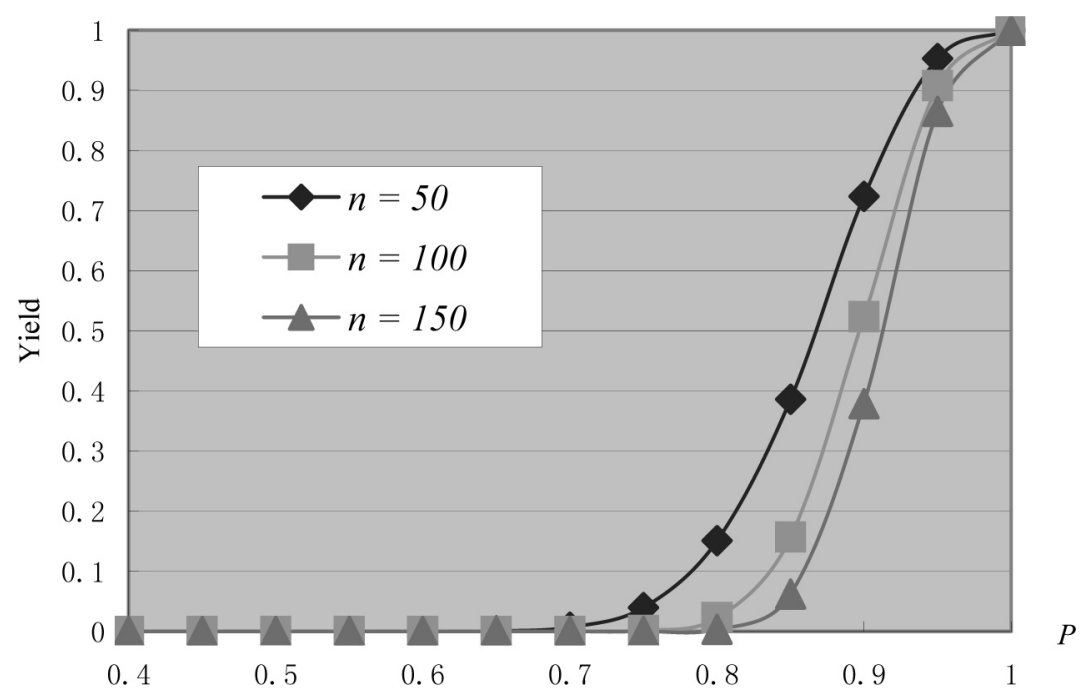

Fig. 14. Estimated yield for $D T M B(2,6)$.

The simulation results on yield estimation for $\operatorname{DTMB}(2,6)$ arrays with different sizes are shown in Figure 14. A similar analytical modeling method can also be applied to $\operatorname{DTMB}(3,6)$ designs as shown in Figure 15 ; their yields can be estimated by

$$
Y_{D T M B(3,6)}=Y_{C}^{n / 6}=\left(p^{9}+9 p^{8}(1-p)+36 p^{7}(1-p)^{2}+84 p^{8}(1-p)\right)^{n / 6} .
$$




\section{$\operatorname{DTMB}(3,6)$ Cluster Graph Model}

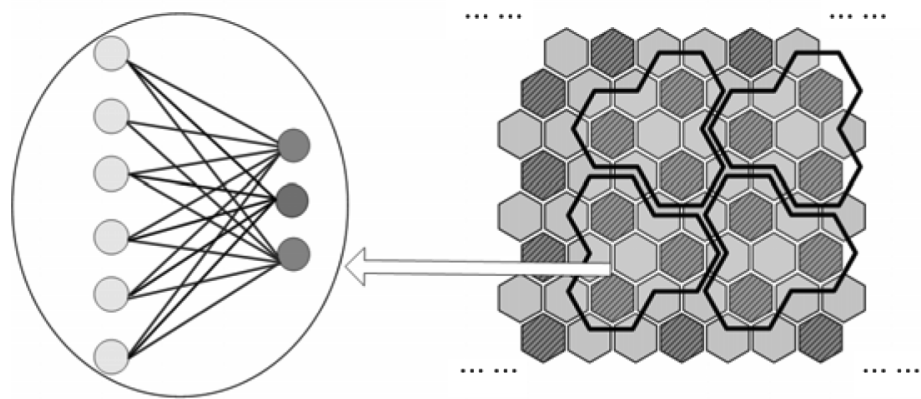

(a)

(b)

Fig. 15. (a) Graph model for a $\operatorname{DTMB}(3,6)$ cluster; (b) illustration of clusters for $\operatorname{DTMB}(3,6)$ array.

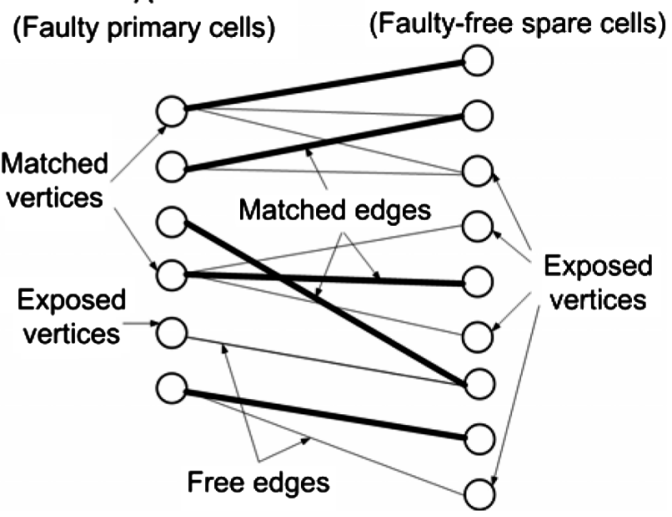

Fig. 16. A maximal bipartite matching graph model.

We can also estimate the yield for a defect-tolerant design using Monte-Carlo simulation. During each run of the simulation, the cells in the microfluidic array, including both primary and spare cells, are randomly chosen to fail with probability $p$. We then check if these defects can be tolerated via local reconfiguration based on the interstitial spare cells. This checking procedure is based on a graph matching approach as described in the following.

We develop a bipartite graph model to represent the relationship between faulty and spare cells in the microfluidic array. A bipartite graph $B G(A, B, E)$ is a graph whose nodes can be partitioned into two sets, $A$ and $B$, and each edge in $E$ has one node in $A$ and one node in $B$ [Su et al. 2003]. In our model, nodes in $A$ represent the faulty primary cells in the microfluidic array, while nodes in $B$ denote the fault-free spare cells. An edge exists from a node $a$ in $A$ to a node $b$ in $B$ if and only if the faulty primary cell represented by $a$ is physically adjacent to the spare cell represented by $b$. An example of a bipartite graph model is shown in Figure 16. A matching $M$ of a bipartite graph $B G(A, B, E)$ is 


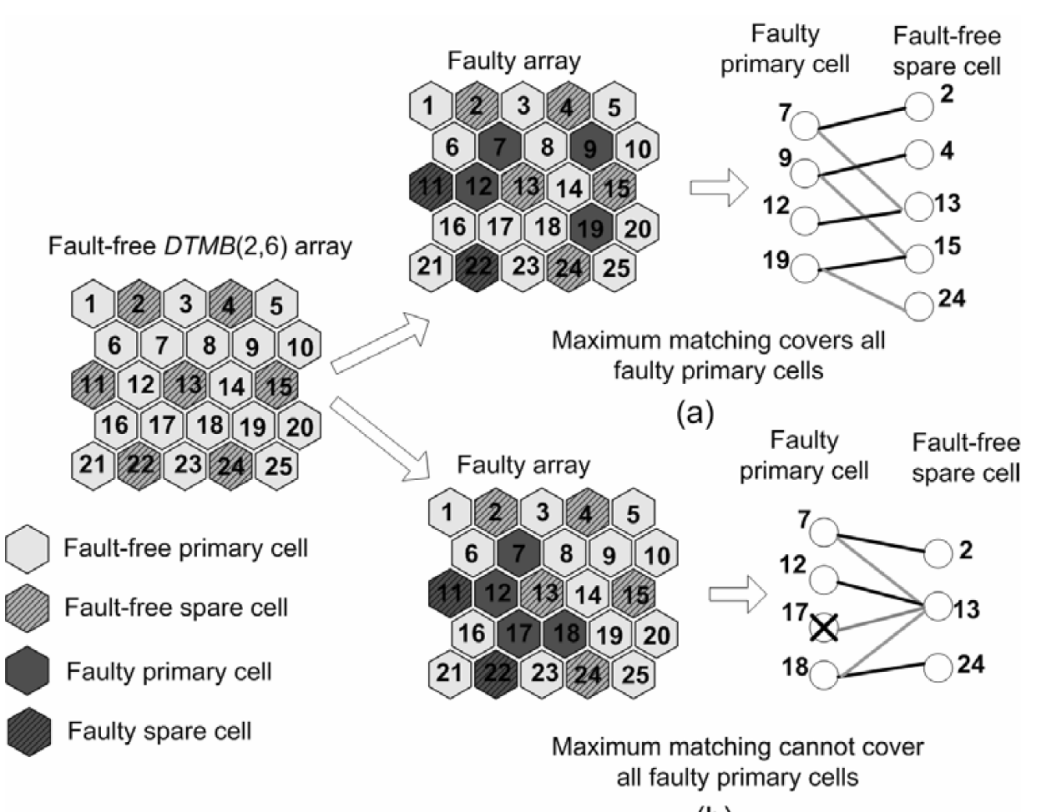

(b)

Fig. 17. Example of using maximal bipartite matching model to determine reconfigurability.

a subset of the edges ( $M \subseteq E$ ) with the property that no two edges of $M$ share the same node. Edges in $M$ are called matched edges, while the other edges are free. If $[a, b]$ is a matched edge, then $a$ is the mate of $b$. Nodes that are not incident upon any matched edge are celled exposed vertices, while the others are called matched vertices. A path $P t=\left[u_{1}, u_{2}, \ldots, u_{k}\right]$, where $u_{i} \in A \cup B$, is called alternating if $\left[u_{1}, u_{2}\right],\left[u_{3}, u_{4}\right], \ldots\left[u_{2 j-1}, u_{2 j}\right], \ldots$ are free whereas $\left[u_{2}\right.$, $\left.u_{3}\right],\left[u_{4}, u_{5}\right], \ldots\left[u_{2 j}, \mathrm{u}_{2 j+1}\right], \ldots$ are matched. We further call an alternating path $P t=\left[u_{1}, u_{2}, \ldots, u_{k}\right]$ an augmenting path if both $u_{1}$ and $u_{k}$ are exposed vertices.

A maximal matching for this bipartite graph model can be obtained using well-known techniques that are based on a theorem stated as follows:

THEOREM 1. A matching $M$ in a graph is maximum if and only if there is no augmenting path in this graph with respect to $M$ [Asratian et al. 1998].

The key idea underlying the algorithm is to start with any matching (e.g., the empty one), and repeatedly search for augmenting paths to augment the matching. The search stops when there is no augmenting path, and then a maximum matching $M_{\max }$ is found. If this maximal matching $M_{\max }$ covers all nodes in $A$, it implies that all faulty cells can be replaced by their adjacent fault-free spare cells through local reconfiguration; an example is shown in Figure 17(a). Otherwise, this microfluidic biochip cannot be reconfigured as shown in Figure 17(b). After a large number of simulation runs, the yield of this microfluidic array is determined from the proportion of successful reconfigurations. The pseudocode for the proposed matching-based Monte-Carlo method is shown in Figure 18. The simulation results for $\operatorname{DTMB}(2,6), \operatorname{DTMB}(3,6)$ and $\operatorname{DTMB}(4,4)$ by the 


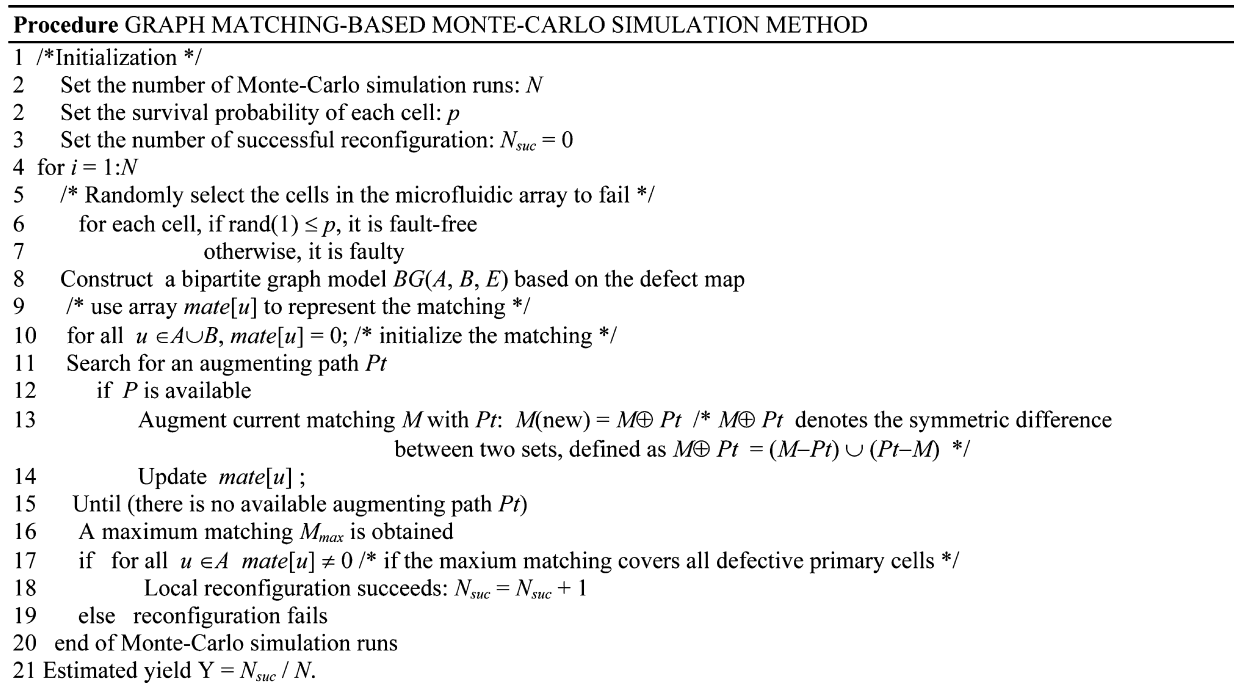

Fig. 18. Pseudocode of Monte-Carlo simulation method for yield estimation.
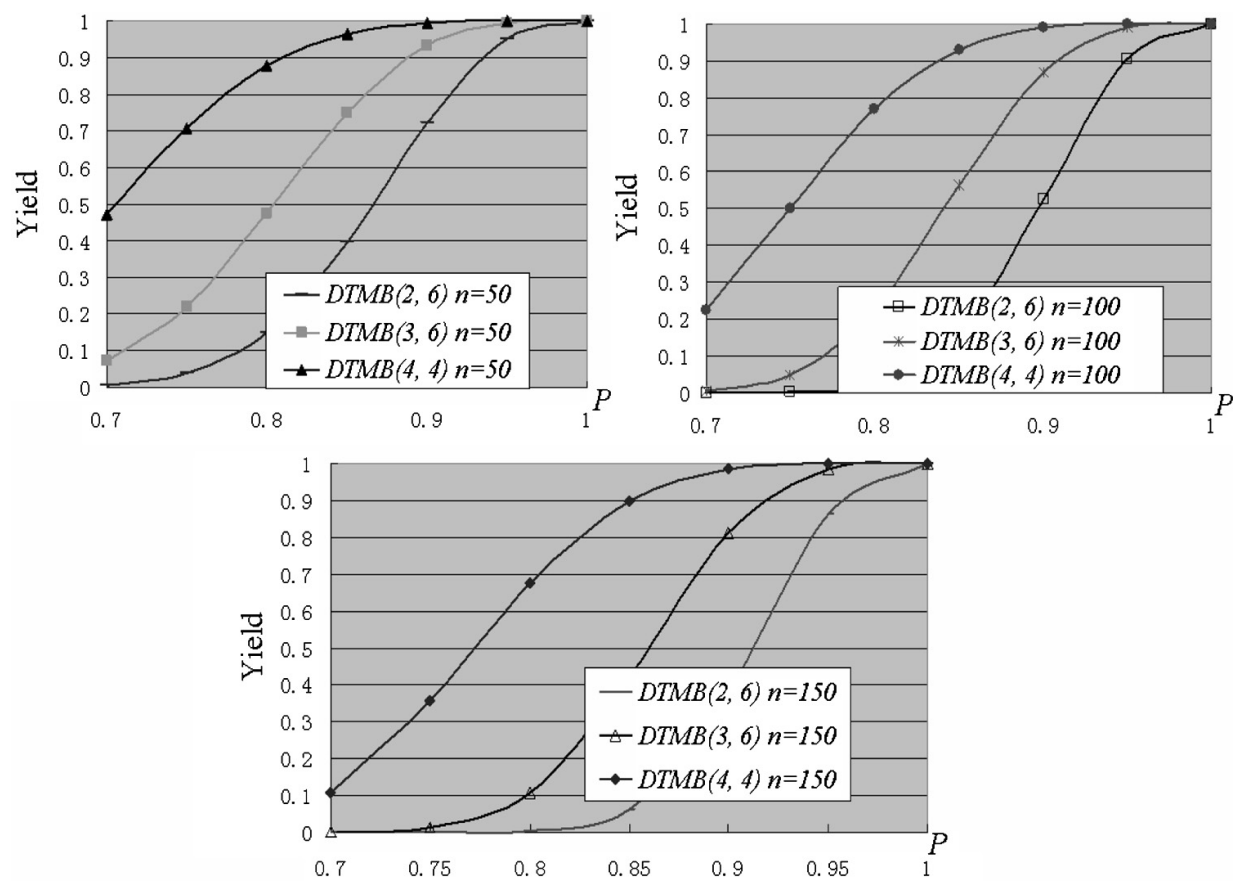

Fig. 19. Yield estimation for $D T M B(2,6), D T M B(3,6)$ and $D T M B(4,4)$.

Monte-Carlo method are shown in Figure 19 where $n$ is the number of primary cells.

We also compare the yield estimation obtained using analytical modeling and Monte-Carlo simulation methods; an example of the comparison for $\operatorname{DTMB}(2,6)$ 


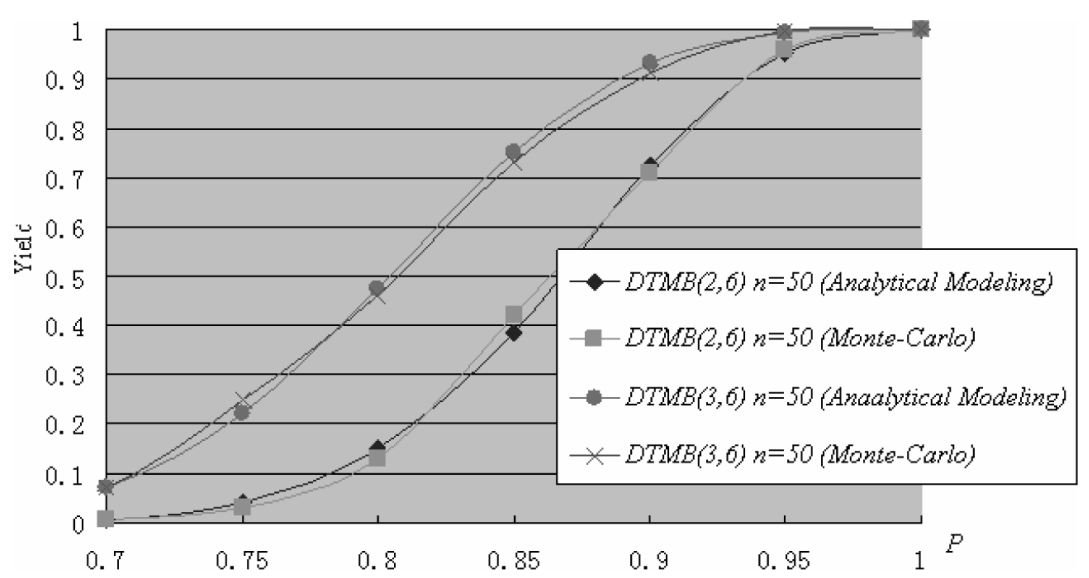

Fig. 20. Comparison of yield estimation by two different methods.

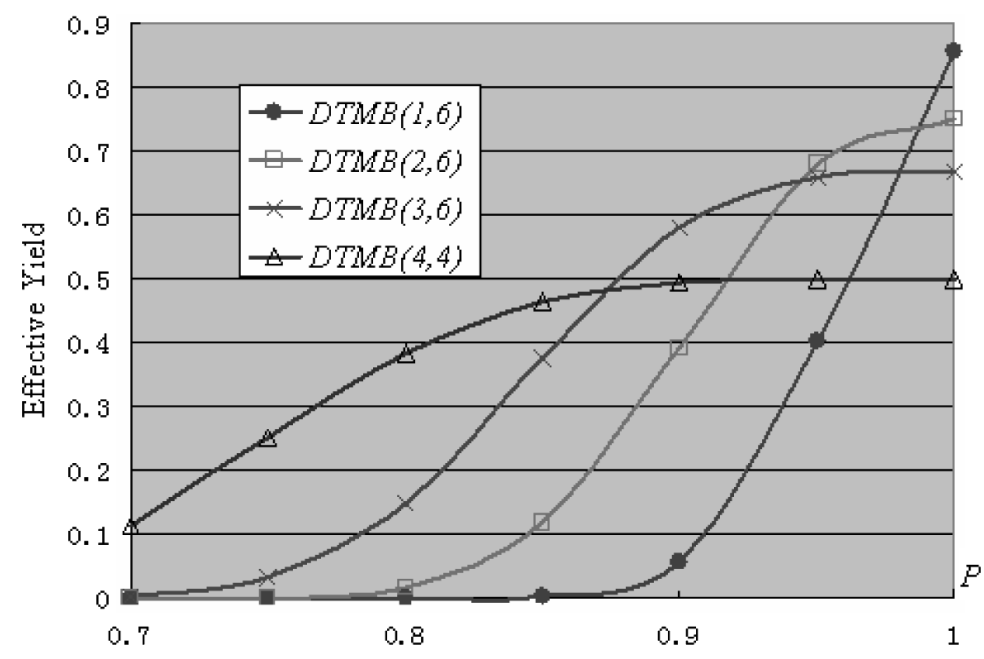

Fig. 21. Effective yield for different levels of redundancy.

and $\operatorname{DTMB}(3,6)$ designs is shown in Figure 20 which shows that the two estimates are close to each other.

From Figure 19, it is clear that a higher level of redundancy leads to a higher yield. However, adding more redundant cells increases the array area and thereby manufacturing cost. To measure yield enhancement relative to the increased array size, we define the effective yield $E Y$ as:

$$
E Y=Y \times(n / N)=Y /(1+R R),
$$

where $n$ is the number of primary cells, and $N$ is the total number of cells in the microfluidic array. The parameter $E Y$ represents the trade-off between yield enhancement and the increase in manufacturing cost. The variation of $E Y$ with $p$ for different redundancy levels is shown in Figure 21. The number of primary cells is set to 100 . As expected, the results show that a microfluidic structure with the higher level of redundancy, such as $\operatorname{DTMB}(4,4)$, is suitable 
for small values of $p$. On the other hand, a lower level of redundancy, such as $\operatorname{DTMB}(1,6)$ or $\operatorname{DTMB}(2,6)$, should be used when $p$ is relatively high.

Note that the yield analysis discussed thus far assumes that all primary cells in the microfluidic array are used in the bioassay. However, in many practical biochip applications, not all primary cells are utilized by bioassays. The unused primary cells need not to be reconfigured even if they are defective. Thus a higher value of the yield can be obtained if the array utilization is also considered; we illustrate this issue with a real-life example in the next section. We can view the yield analysis results in this section as application-independent indexes which are used as a guideline for defect-tolerant biochip designs.

\section{EXAMPLE: MULTIPLEXED IN-VITRO DIAGNOSTICS}

In this section, we evaluate the proposed defect-tolerant design by applying it to a digital microfluidics-based biochip used for multiplexed biomedical assays. The in-vitro measurement of glucose and other metabolites, such as lactate, glutamate and pyruvate, in human physiological fluids plays a critical role in clinical diagnosis of metabolic disorders. For example, the change of regular metabolic parameters in the patient's blood can signal organ damage or dysfunction prior to observable microscopic cellular damages or other symptoms. Recently, the feasibility of performing a colorimetric enzyme-kinetic glucose assay on a digital microfluidic biochip has been successfully demonstrated in experiments [Srinivasan et al. 2004].

The glucose assay performed on the biochip is based on Trinder's reaction, a colorimetric enzyme-based method [Trinder 1969]. The enzymatic reactions involved in the assay are:

$$
\begin{array}{r}
\text { Glucose }+\mathrm{H}_{2} \mathrm{O}+\mathrm{O}_{2} \stackrel{\text { GlucoseOxidase }}{\longrightarrow} \text { Gluconic Acid }+\mathrm{H}_{2} \mathrm{O}_{2}, \\
2 \mathrm{H}_{2} \mathrm{O}_{2}+4-\mathrm{AAP}+\mathrm{TOPS} \stackrel{\text { Peroxidase }}{\longrightarrow} \text { Quinoneimine }+4 \mathrm{H}_{2} \mathrm{O} .
\end{array}
$$

In the presence of Glucose oxidase, glucose can be enzymatically oxidized to gluconic acid and hydrogen peroxide. Then, in the presence of peroxidase, the hydrogen peroxide reacts with 4-amino antipyrine (4-AAP) and N-ethyl-Nsulfopropyl-m-toluidine (TOPS) to form violet-colored quinoneimine which has an absorbance peak at 545nm. Based on Trinder's reaction, a complete glucose assay can be performed following three steps, namely, transportation, mixing, and optical detection as shown in Figure 22. A sample droplet containing glucose and a reagent droplet containing glucose oxidase, peroxidase, 4-AAP, and TOPS, are dispensed into the microfluidic array from their respective droplet sources. They are then transported towards a mixer where the sample and the reagent droplets are mixed. The mixed droplet is transported onto a transparent electrode in order to observe the absorbance of the products of the enzymatic reaction. Absorbance measurements are performed with a green LED and a photodiode. The glucose concentration can be measured from the absorbance which is related to the concentration of colored quinoneimine in the droplet. Besides glucose assays, other metabolites such as lactate, glutamate, and pyruvate have been detected in a digital microfluidic biochip recently. In 


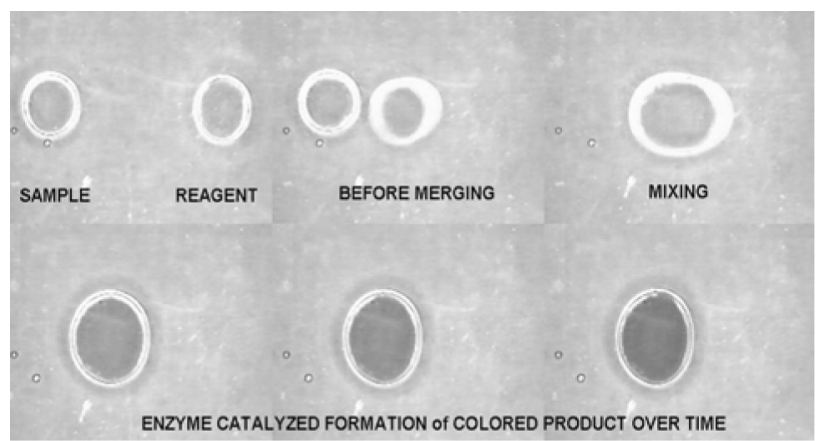

Fig. 22. Photos of different steps of a glucose assay carried out on a digital microfluidic biochip [Srinivasan et al. 2004].

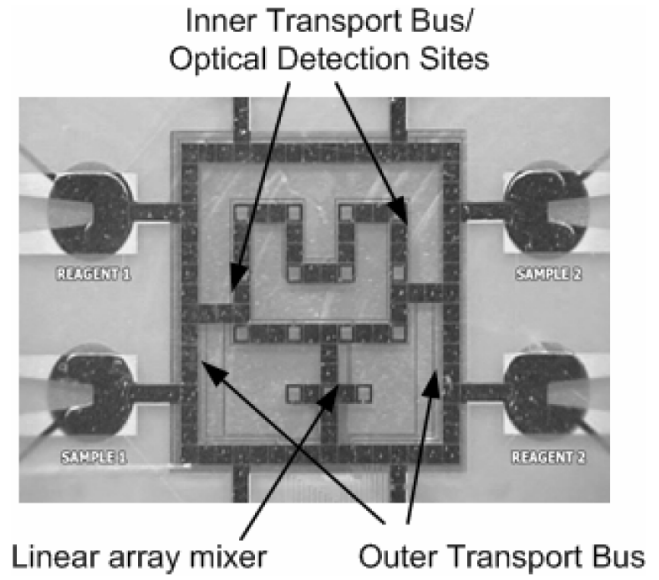

Fig. 23. Fabricated biochip used for multiplexed bioassays.

addition to current metabolites, a number of reagents can be added onto a chip to enable a multiplexed in-vitro diagnostics platform on different human physiological fluids. Figure 23 shows a recently fabricated microfluidic biochip used for multiplexed biomedical assays [Srinivasan et al. 2004]. In this biochip with square electrodes, SAMPLE1 and SAMPLE2 contain glucose and REAGENT1 and REAGENT2 contain the reagents.

In this first design and demonstration, only cells used for the bioassays were fabricated; no spare cells were included in the array. Thus, even if one arbitrary cell in this biochip becomes faulty due to a manufacturing defect, this failure cannot be avoided by reconfiguration, and the fabricated biochip has to be discarded. Consequently, the yield for this biochip design is very low. It is only 0.3378 even if the survival probability of a single cell is as high as 0.99 . Such low yield makes the first biochip design unsuitable for future mass fabrication and use in clinic diagnostics.

In order to improve the yield, we use a defect-tolerant design with interstitial redundancy as described in Section 7. To facilitate the comparison, the topological structure of primary cells in the first design is directly mapped to a 


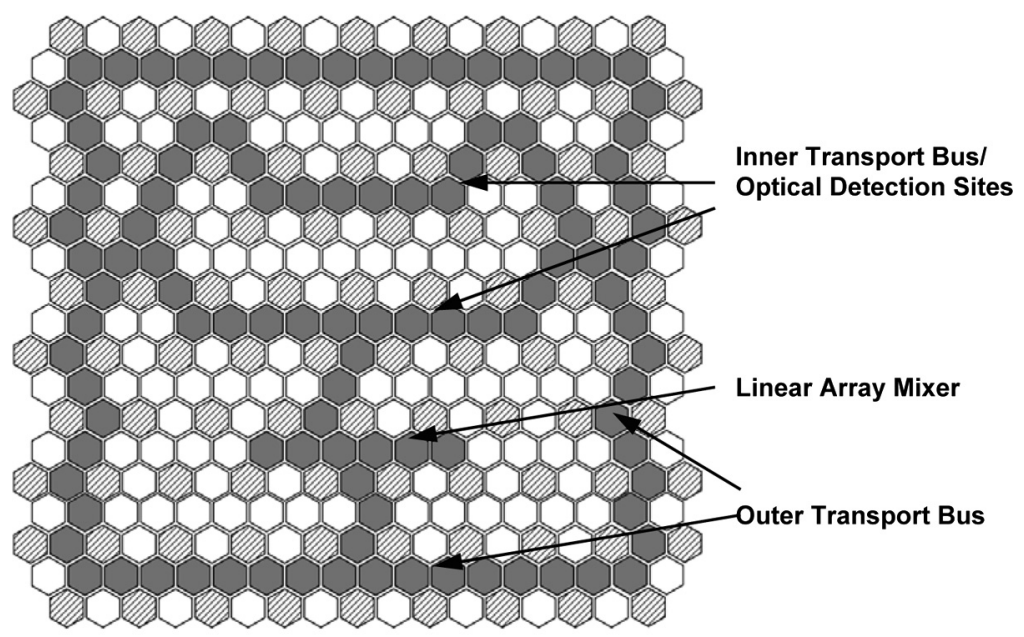

Spare cell $\bigcirc$ Unused Primary cell

Primary cell used in biomedical assays

Fig. 24. A defect-tolerant design based on $\operatorname{DTMB}(2,6)$.

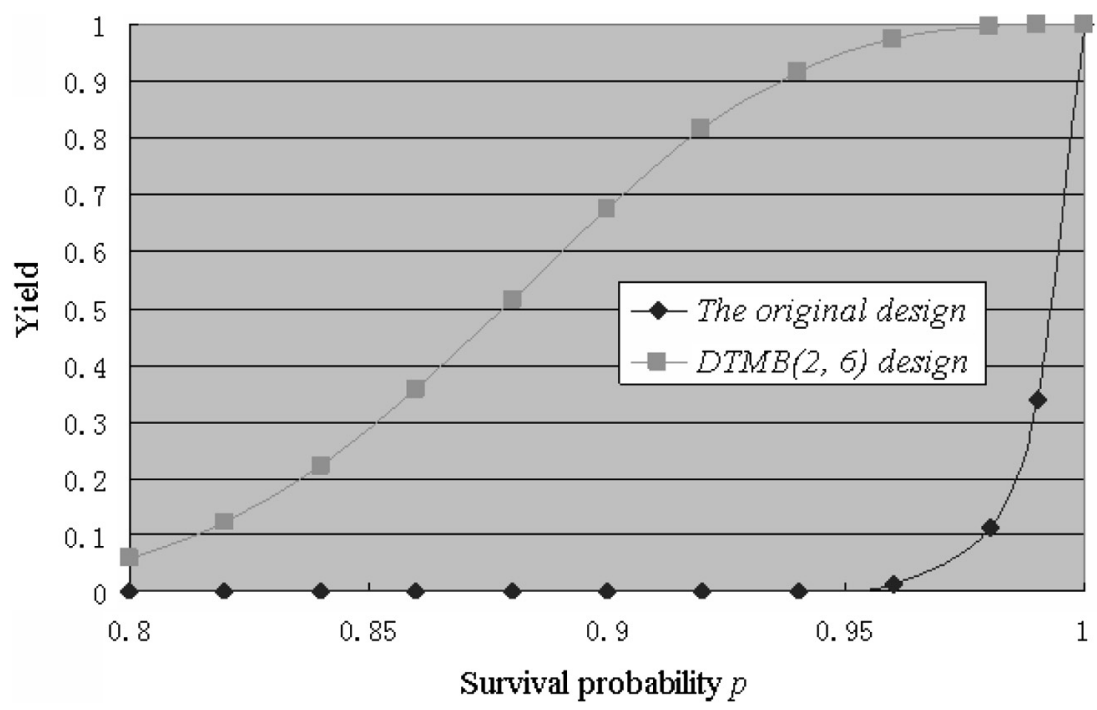

Fig. 25. Estimated yield enhancement of $\operatorname{DTMB}(2,6)$ design.

$\operatorname{DTMB}(2,6)$ design. (Note that here $\operatorname{DTMB}(2,6)$ is chosen for ease of illustration. The following analysis can be easily extended to other defect-tolerant arrays such as $\operatorname{DTMB}(1,6)$ and $\operatorname{DTMB}(3,6)$ designs.) The new defect-tolerant design has the same number of primary cells used for multiplexed biomedical assays as the original design, see Figure 24 . There are 252 primary cells (108 of them used in assays) and 91 spare cells in this defect-tolerant biochip. Figure 25 shows 


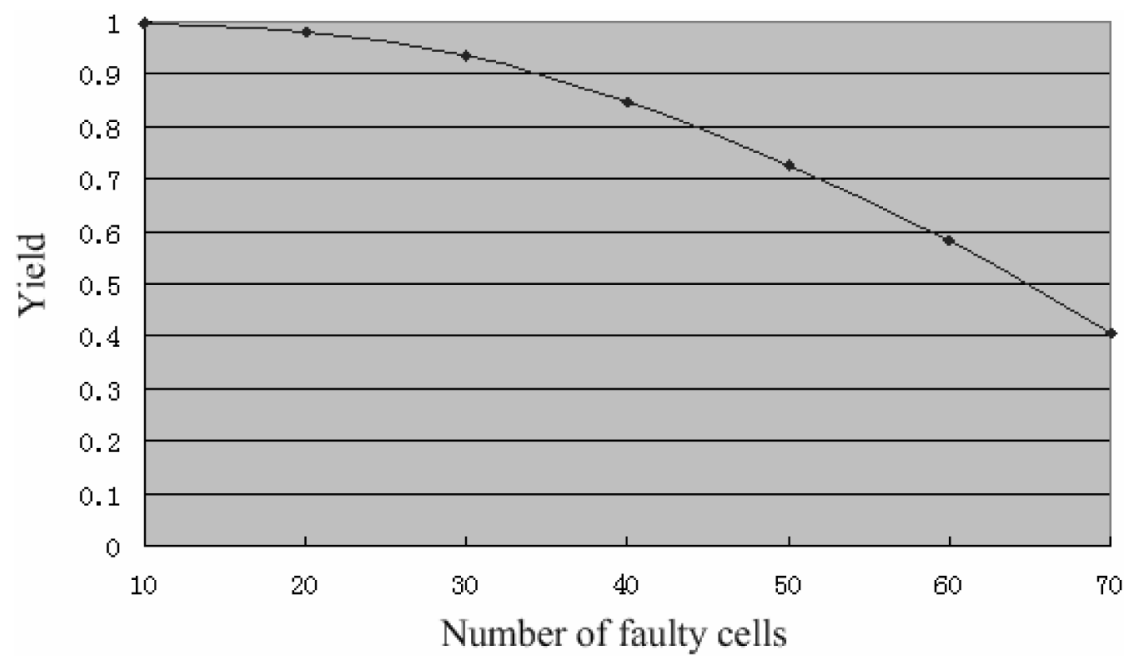

Fig. 26. Yield estimation for the $D T M B(2,6)$-based design in the presence of multiple defects.

the estimated yield of this defect-tolerant design by the Monte-Carlo method for different values of survival probability $p$ of a single hexagonal electrode. Compared to the original design, the $\operatorname{DTMB}(2,6)$ design leads to a significantly higher yield.

To further illustrate the improvement in yield, we randomly introduce $m$ cell failures, and then apply local reconfiguration to avoid them. The yield in the presence of $m$ failures is obtained through Monte-Carlo simulation. The yield for the different values of $m$ is shown in Figure 26. For up to 35 faults, the redundant design can provide a yield of at least 0.90 . An example of successful reconfiguration in the presence of 10 faulty cells is shown in Figure 27.

In the $\operatorname{DTMB}(2,6)$ design, although there are a total of 252 primary cells in the array, only 108 primary cells are used in the biochemical application. The remaining primary cells can serve as spare cells or they can be used later in a new set of remapped bioassays. This spare design has the advantage of easy droplet motion control since enough spacing between different droplet routes prevents multiple droplets from being unintentionally mixed [Su et al. 2006]. However, we can further map the design into a smaller array as shown in Figure 28. This new packed $\operatorname{DTMB}(2,6)$ design has the same number (i.e., 108) of primary cells used in assay but with a much smaller array size (a total of 196 cells, $43 \%$ less than the previous design). We next estimate the yield for these two different $D T M B(2,6)$ designs, see Figure 29 . Note that the yield for the spare $\operatorname{DTMB}(2,6)$ design is slightly higher than the packed design due to more unused primary cells that need not be reconfigured. However, to take biochip area overhead into account, we further analyze the effective yield for these two designs, that is, $E Y=Y \times(108 / N)$, where $N$ is the total number of cells in the microfluidic array. The simulation results in Figure 30 show that, for a relatively high value of survival probability $p$, the packed $\operatorname{DTMB}(2,6)$ design is more suitable than the spare design when both yield enhancement and increase in manufacturing cost are considered. 

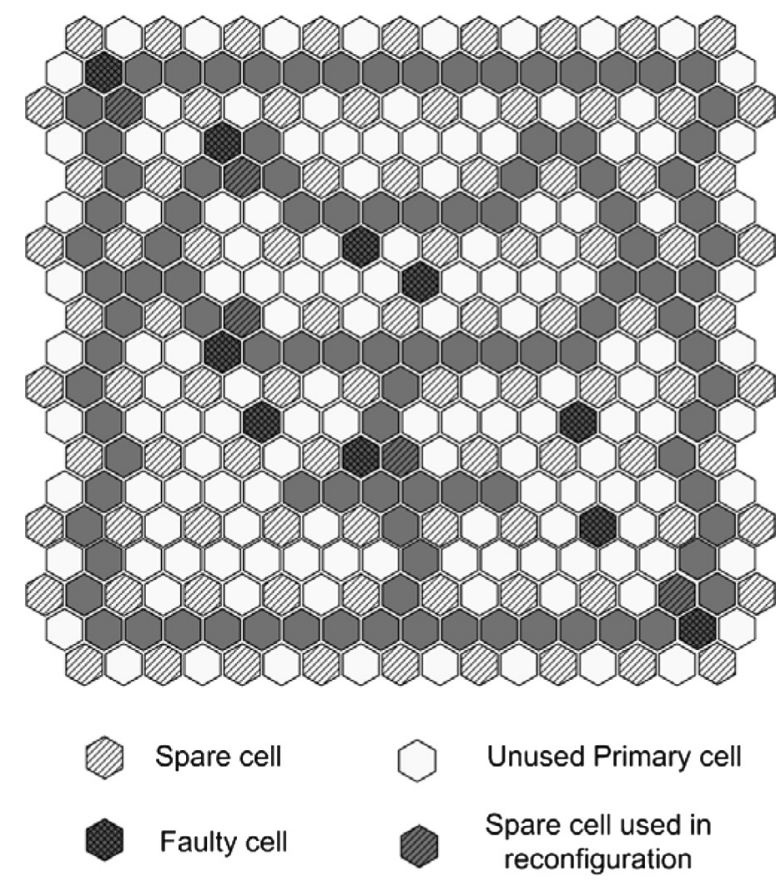

Primary cell used in biomedical assays

Fig. 27. An example of local reconfiguration.

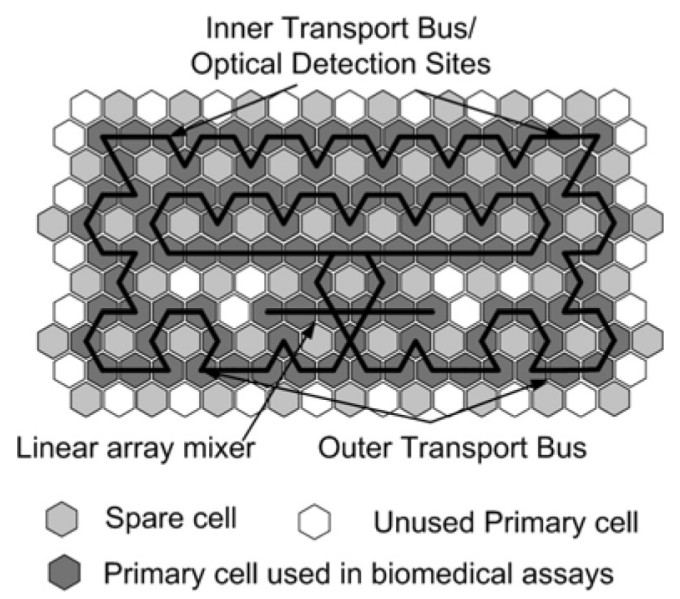

Fig. 28. A more packed defect-tolerant design based on $D T M B(2,6)$.

\section{CONCLUSIONS}

In this article, we have presented a yield enhancement technique for digital microfluidics-based biochips. This technique relies on (i) space redundancy, whereby spare cells are placed in the interstitial sites of the microfluidic array, and (ii) local reconfiguration in which spare cells replace the neighboring 


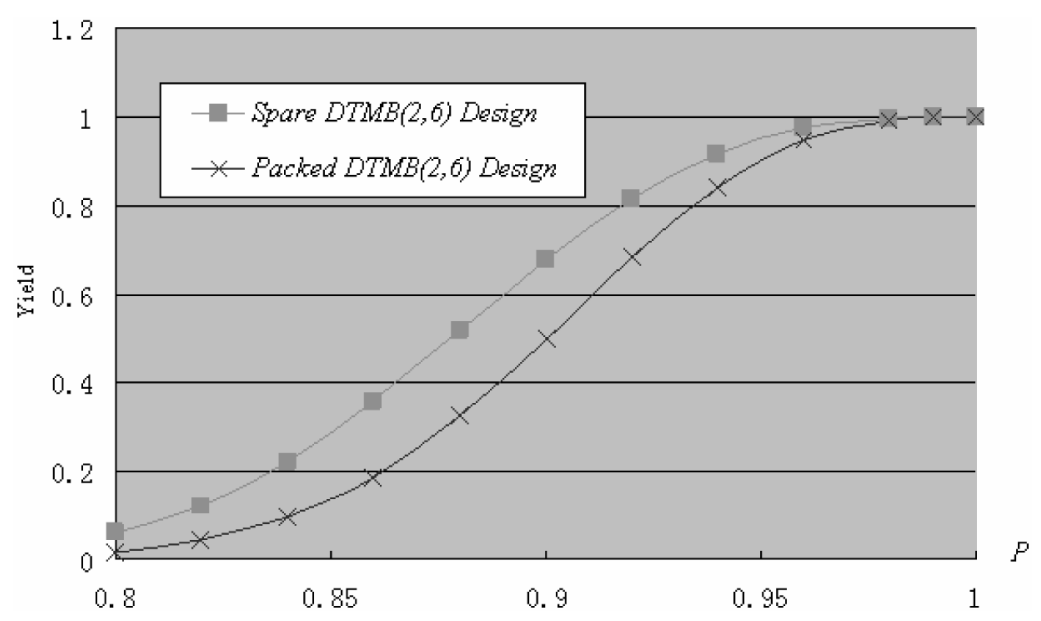

Fig. 29. Yield estimation for the spare and packed $D T M B(2,6)$ designs.

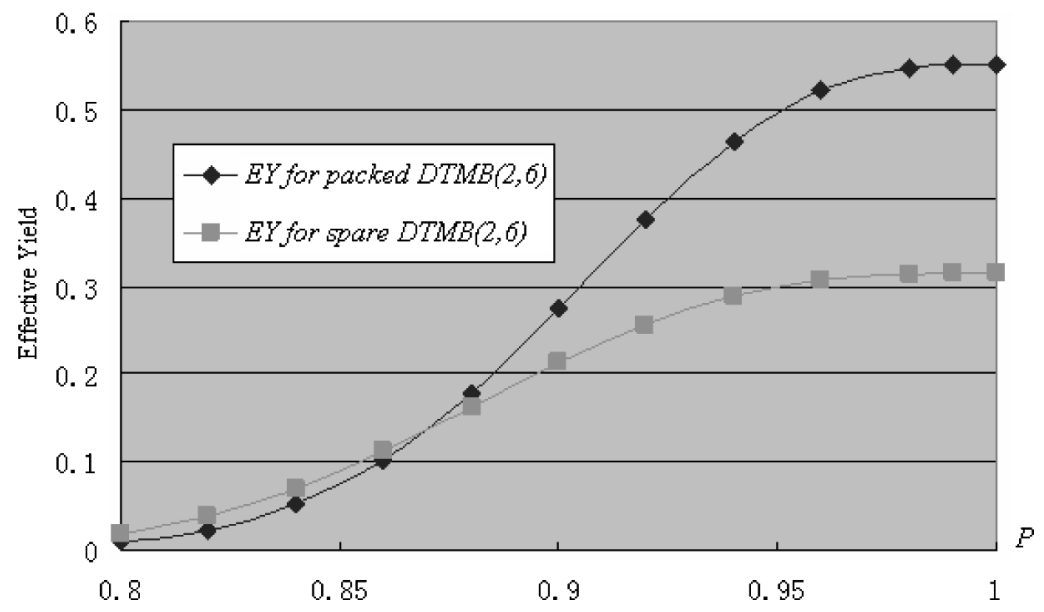

Fig. 30. Effective yield for the spare and packed $D T M B(2,6)$ designs.

faulty cells. The defect-tolerant design has been evaluated for a set of real-life bioassays. Low yield, which is expected to be a consequence of increased area and density of biochips, will be a deterrent to high-volume production and it will increase production cost. The proposed defect-tolerant design approach for yield enhancement will therefore be especially useful for the emerging marketplace.

\section{ACKNOWLEDGMENTS}

We thank Vamsee Pamula of Advanced Liquid Logic (http://www.liquidlogic.com) and William Hwang of Duke University for their help in this work.

\section{REFERENCES}

Asratian, A. Denley, T., and Haeggkvist, R 1998. Bipartite Graphs and Their Applications. Cambridge University Press, NY, NY. 
Dewey, A. Ren, H., AND ZhANG, T. 2000. Behavioral modeling of microelectromechanical systems (MEMS) with statistical performance-variability reduction and sensitivity analysis. IEEE Trans. Circuits and Sys. II 47, 105-113.

Howard, N., TyrRel, A. ANd Allinson, N. 1994. The yield enhancement of Field-Programmable Gate Arrays. IEEE Trans. VLSI Syst. 2, 115-123.

International Technology Roadmap for Semiconductor (ITRS), http://public.itrs.net/Files/ 2003ITRS/Home2003.htm.

JeE, A. And Ferguson, F. J. 1993. Carafe: An inductive fault analysis tool for CMOS VLSI circuits. In Proceedings of the IEEE VLSI Test Symposium. 92-98.

Konen, I. AND Koren, Z. 1998. Defect tolerance in VLSI circuits: techniques and yield analysis, In Proceedings of the IEEE. 86, 1819-1836.

Madou, M. 1997. Fundamentals of Microfabrication. CRC Press, Boca Raton, FL.

Papadimitriou, C. and Steiglitz, K. 1982. Combinatorial Optimization: Algorithms and Complexity. Prentice-Hall, Englewood Cliffs, NJ.

PolLACK, M. 2001. Electrowetting-based microactuation of droplets for digital microfluidics, $\mathrm{PhD}$ thesis, Duke University, Durham, NC.

Pollack, M. G., Shenderov, A. D., AND FAIR, R. B. 2000. Electrowetting-based actuation of liquid droplets for microfluidic applications. Appli. Physics Lett. 77, 1725-1726.

Pollack, M. G., Shenderov, A. D., ANd FAIR, R. B. 2002. Electrowetting-based actuation of droplets for integrated microfluidics. Lab on a Chip 2, 96-101.

SingH, A. 1988. Interstitial redundancy: an area efficient fault tolerance scheme for large area VLSI processor arrays. IEEE Trans. Comput. 37, 1398-1410.

SRinivasan, V., Pamula, V. K., And Fair, R. B. 2004. An integrated digital microfluidic lab-on-a-chip for clinical diagnostics on human physiological fluids. Lab on a Chip 4, 310-315.

Srinivasan, V., Pamula, V. K., Pollack, M. G., and Fair, R. B. 2003. Clinical diagnostics on human whole blood, plasma, serum, urine, saliva, sweat, and tears on a digital microfluidic platform. In Proceedings $\mu$ TAS. 1287-1290.

Su, F. AND Chakrabarty, K. 2005. Design of fault-tolerant and dynamically-reconfigurable microfluidic biochips. In Proceedings on Design, Automation and Test in Europe (DATE) Conference. 1202-1207.

Su, F., Hwang, W., and Chakrabarty, K. 2006. Droplet routing in the synthesis of digital microfluidic biochips. Proceedings on Design, Automation and Test in Europe (DATE) Conference. To appear.

Su, F., Hwang, W., Mukherjee, A., and Chakrabarty, K. 2005. Defect-oriented testing and diagnosis of digital microfluidics-based biochips. In Proceedings of the IEEE International Test Conference.

Su, F., Ozev, S., and Chakrabarty, K. 2003. Testing of droplet-based microelectrofluidic systems. In Proceedings of the IEEE International Test Conference. 1192-1200.

Su, F., Ozev, S., and Chakrabarty, K. 2005. Ensuring the operational health of droplet-based microelectrofluidic biosensor systems. IEEE Sensors 5, 763-773.

Su, F., Ozev, S., And Chakrabarty, K. 2004. Concurrent testing of droplet-based microfluidic systems for multiplexed biomedical assays. In Proceedings of the IEEE International Test Conference. 883-892.

TRINDER, P. 1969. Determination of glucose in blood using glucose oxidase with an alternative oxygen acceptor. Ann. Clinical Biochem. 6, 24-27.

VERPOORTE, E., AND De, RoOIJ, N. F. 2003. Microfluidics meets MEMS. In Proceedings of the IEEE. 91, 930-953.

Received October 2005; revised January, March 2006; accepted April 2006 